\title{
A Case of Anterior Cranial Fossa Dural Case Report Arteriovenous Fistula with Transient Ocular Movement Disorder after Embolization
}

Yuko Tanaka, Tomomi Yoshiyama, Akira Nishiyama, Arisa Umesaki, Takato Nakajou, Hiroaki Matsumoto, and Tomoaki Terada

Objective: We report a case of anterior cranial fossa dural arteriovenous fistula (dAVF) in which ocular movement was impaired after Onyx embolization from the ophthalmic artery (OphA).

Case Presentation: A 76-year-old male was admitted to our hospital for treatment of an incidentally found anterior cranial fossa dAVF. Onyx was injected from the right anterior ethmoidal artery (AEA) to close the shunt. Onyx refluxed to the third portion of the OphA to make a plug, but was unable to reach the venous side beyond the shunt; therefore, a small shunt remained. Although his visual acuity and field were normal, vertical diplopia developed after embolization and disappeared 1 month later. Diplopia worsened when the patient tilted his head to the right. Neuro-ophthalmological examination confirmed right superior oblique muscle impairment. The cause of diplopia was considered to be ischemic injury of the superior oblique muscle associated with embolization of the AEA, which provides nutrients to the superior oblique muscle and trochlear nerve.

Conclusion: Embolization from the OphA beyond the third portion may cause external ophthalmoplegia, although it may heal spontaneously.

Keywords d dural arteriovenous fistula, complication, Onyx, superior oblique muscle paralysis, anterior cranial fossa dural arteriovenous fistula

\section{Introduction}

In cases of anterior cranial fossa dural arteriovenous fistulae (dAVF), the main feeder is usually a small branch arising from the ophthalmic artery (OphA). To achieve angiographical cure by embolization, it is necessary to embolize the dAVF via the feeder from OphA. However, embolization from OphA could lead to visual disturbance related to occlusion of the central retinal artery (CRA). ${ }^{1,2)}$ Embryologically, the CRA is a branch of primitive OphA, but the distal from the second portion of the OphA is

$\overline{\text { Department of Neurosurgery, Showa University Fujigaoka }}$ Hospital, Yokohama, Kanagawa, Japan

Received: March 9, 2019; Accepted: March 5, 2020

Corresponding author: Yuko Tanaka. Department of Neurosurgery, Showa University Fujigaoka Hospital, 1-30 Fujigaoka, Aoba-ku, Yokohama, Kanagawa 227-8501, Japan

Email: ukopin0825@gmail.com

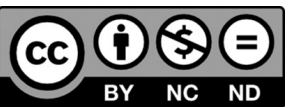

This work is licensed under a Creative Commons Attribution-NonCommercialNoDerivatives International License.

C2020 The Japanese Society for Neuroendovascular Therapy originally a stapedial artery. ${ }^{3)}$ When performing embolization through the OphA, it is important to advance a microcatheter to the periphery to the second portion beyond the CRA. It has been considered to be relatively safe to embolize the lesion beyond the third portion of the OphA. $\left.{ }^{3}\right)$

Here, we report a case of anterior cranial fossa dAVF showing a rare complication of impairment of extraocular movement developing after transarterial embolization (TAE) with Onyx Liquid Embolic System (Medtronic, Minneapolis, MN, USA) (Onyx) via the anterior ethmoidal artery (AEA).

\section{Case Presentation}

A 76-year-old male was admitted to our department for the treatment of asymptomatic anterior cranial fossa dAVF. The dAVF was incidentally found on magnetic resonance imaging (MRI) performed to evaluate asymptomatic right internal carotid artery (ICA) stenosis. He had medical history of abdominal aortic aneurysm treated by endovascular aortic repair, pulmonary emphysema, dyslipidemia, and angina pectoris.

On admission, he had no neurological deficit. 

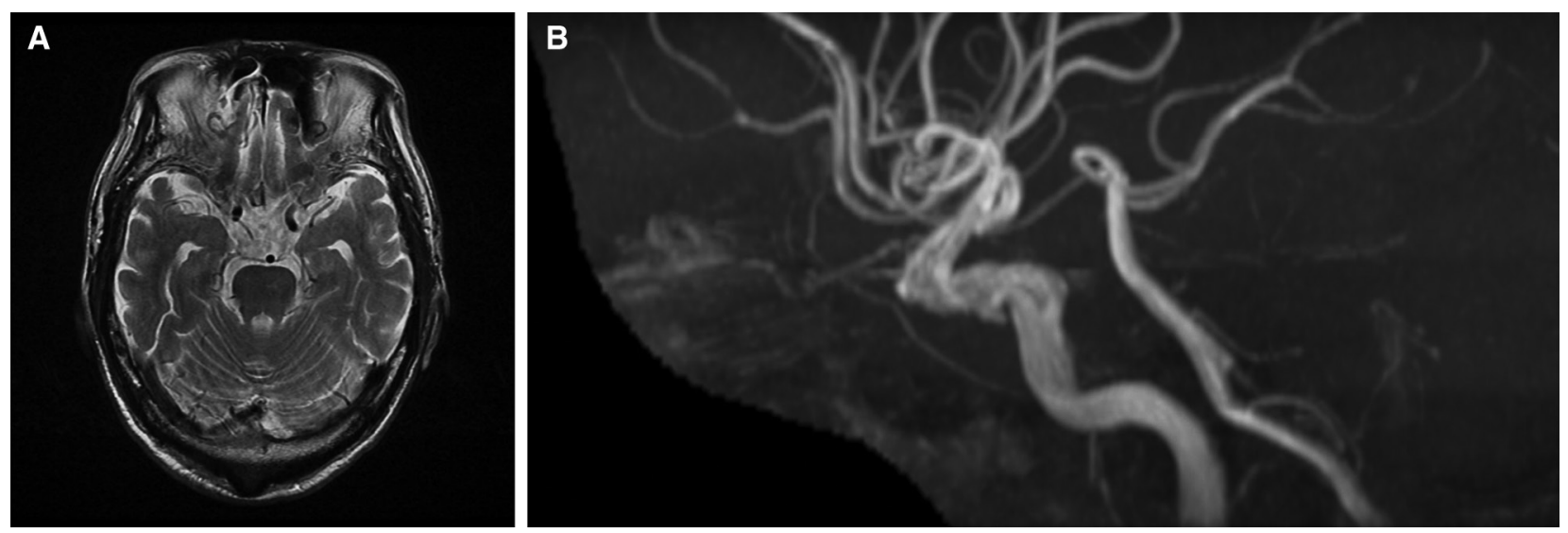

Fig. 1 Cephalic MRI. (A) T2 axial: there was a flow void at the right anterior cranial base. (B) MRA: there was a tortuous/dilated blood flow signal involving the periphery of the OphA to the SSS. 3D TOF MRA revealed tortuous and dilated vascular signals from the OphA at the anterior fossa. MRA: magnetic resonance angiography; OphA: ophthalmic artery; SSS: superior sagittal sinus; TOF: time of flight
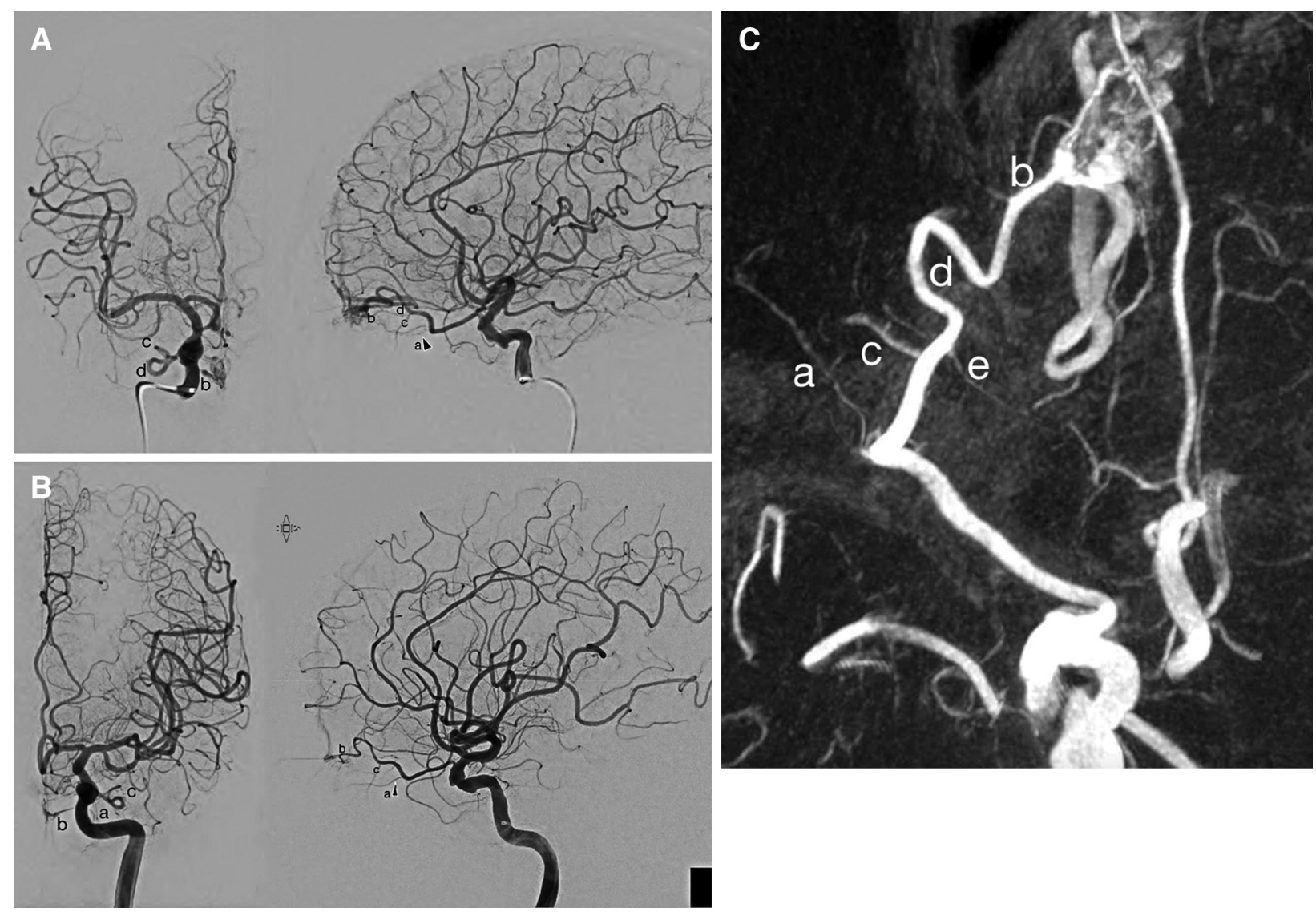

Fig. 2 Preoperative cerebral angiography. (A) Right internal carotid angiography (lateral view). (B) Left internal carotid angiography (lateral view). (C) 3D-DSA (right ICA) (MIP-rearranged image, LAO: $69^{\circ}$, Caudal: $73^{\circ}$ ). (a) Central retinal artery/posterior ciliary artery, (b) anterior ethmoidal artery, (c) inferior medial muscular branch, (d) third portion of the OphA, and (e) PEA. DSA: digital subtraction angiography; ICA: internal carotid artery; LAO: left anterior oblique; OphA: ophthalmic artery; PEA: posterior ethmoidal artery

T2-weighted MRI images demonstrated flow voids at the right anterior cranial base. Maximum intensity projection (MIP) and source images of 3D time of flight (TOF) magnetic resonance angiography (MRA) revealed tortuous and dilated vascular signals from the OphA at the anterior fossa (Fig. 1).
Cerebral angiography of the ICA revealed an anterior cranial fossa dAVF fed by the bilateral AEAs. The shunt point was located in the dura mater of the cribriform plate. The dAVF was drained via two drainage routes, the enlarged olfactory vein continued to an ascending frontal vein to the 


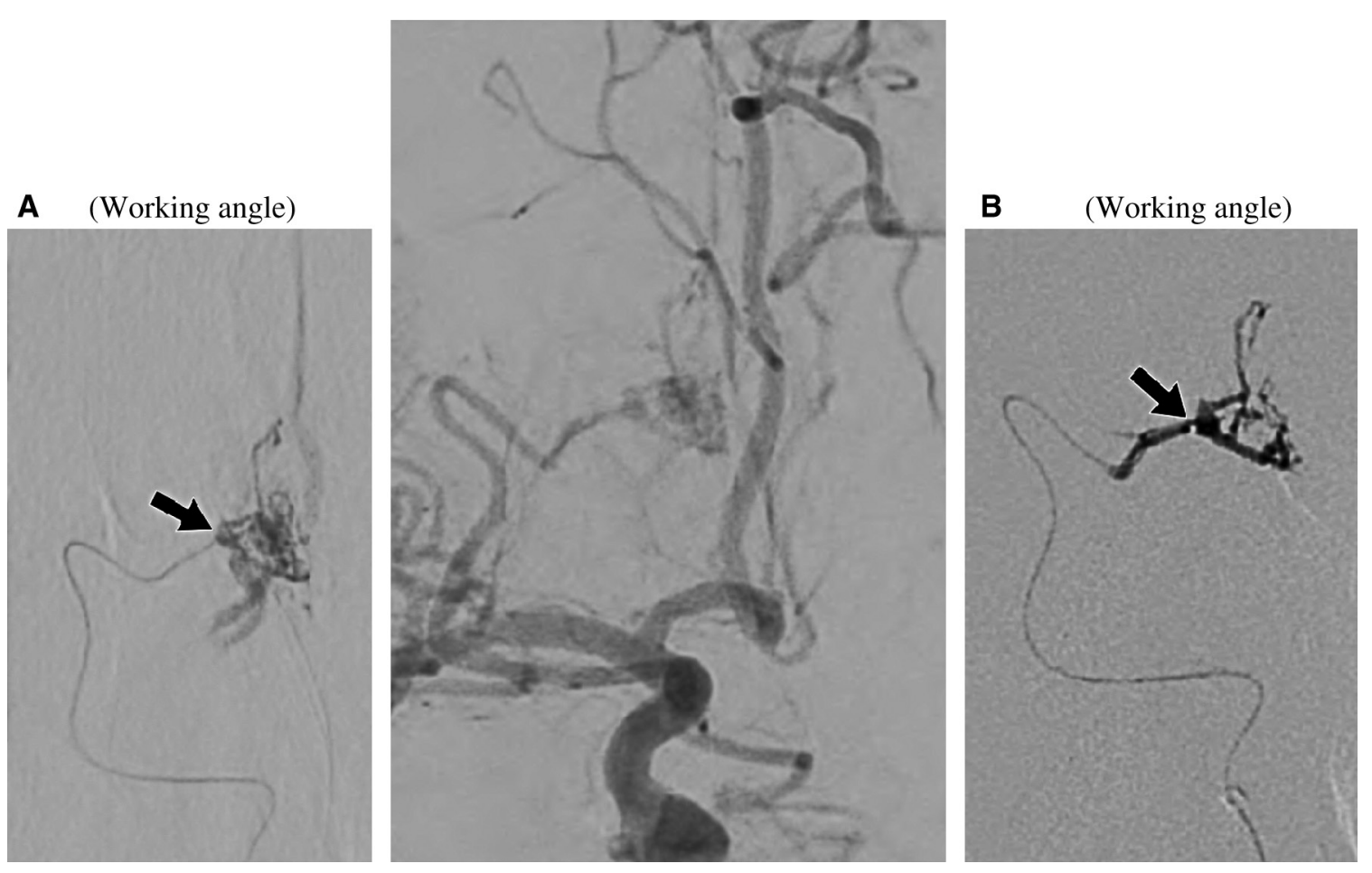

Fig. 3 Intraoperative angiography. Selective angiography through a Marathon guided from the right anterior ethmoidal artery to an area adjacent to the shunt point. The arrow indicates the catheter end: (A) working angle; LAO: $9^{\circ}$, Caudal: $\left.30^{\circ}\right)(B)$ Onyx cast (working angle).

superior sagittal sinus (SSS) and another small ascending frontal vein directly continued from the dAVF to the SSS.

Bilateral external carotid angiography showed no shunt (Fig. 2).

As the risk of intracranial hemorrhage due to the anterior cranial fossa dAVF with a dilated drainage vein was high, we decided to perform transarterial embolization (TAE) through the right AEA to reduce the future risk of hemorrhage.

Under general anesthesia, a 7-F sheath was inserted through the right femoral artery, and a 4-F sheath was inserted through the left side, and heparin was then administered systemically.

After placement of a 7-F Fubuki (hard type) (Asahi Intecc, Co., Ltd., Aichi, Japan) into the cervical portion of the right ICA, a 4.2-F Fubuki (Asahi Intecc, Co., Ltd.) was advanced up to the cavernous portion of the ICA. A 1.3-F microcatheter (Marathon, Medtronic, Minneapolis, MN, USA) was inserted into the AEA through the OphA, and advanced near the shunt point. Through the Marathon, Onyx 18 was injected with the plug and push technique; however, it did not reach the shunt point. Onyx reflux from the AEA back to the third portion of the OphA was noted (Fig. 3). The right internal carotid angiography confirmed the disappearance of shunt flow and drainage to the cortical vein, but small shunt flow from the AEA to the SSS remained on the left internal carotid angiography
(Fig. 4). The feeder was markedly tortuous, and further reflux was considered to make it impossible to remove the Marathon. Therefore, onyx injection was discontinued and the Marathon was removed (total Onyx 18 volume: $0.2 \mathrm{~mL}$ ). On the right internal carotid angiography after embolization, the posterior ethmoidal artery (PEA) and muscular branch were visualized. The third portion of the right OphA distal to the (origin of the PEA) was occluded.

After embolization, the superior frontal vein was not visualized, which indicating the decrease of the risk of hemorrhage. At this point, the procedure was stopped without additional embolization from the left AEA.

Postoperative computed tomography (CT) and conebeam CT confirmed an Onyx cast, which was consistent with the OphA to AEA, located between the intraorbital area and the anterior ethmoidal foramen (Fig. 5).

After embolization, his visual acuity and field were normal, but vertical diplopia developed. It deteriorated when his head was tilted to the right, whereas it disappeared when tilting to the left (Bielschowsky's sign was positive), suggesting paralysis of the right superior oblique muscle. Hess test also confirmed slight paralysis of the right superior oblique muscle (Fig. 6). There were no new abnormal findings on contrast-enhanced MRI or abnormalities, including the extrinsic ocular muscle. Considering the 

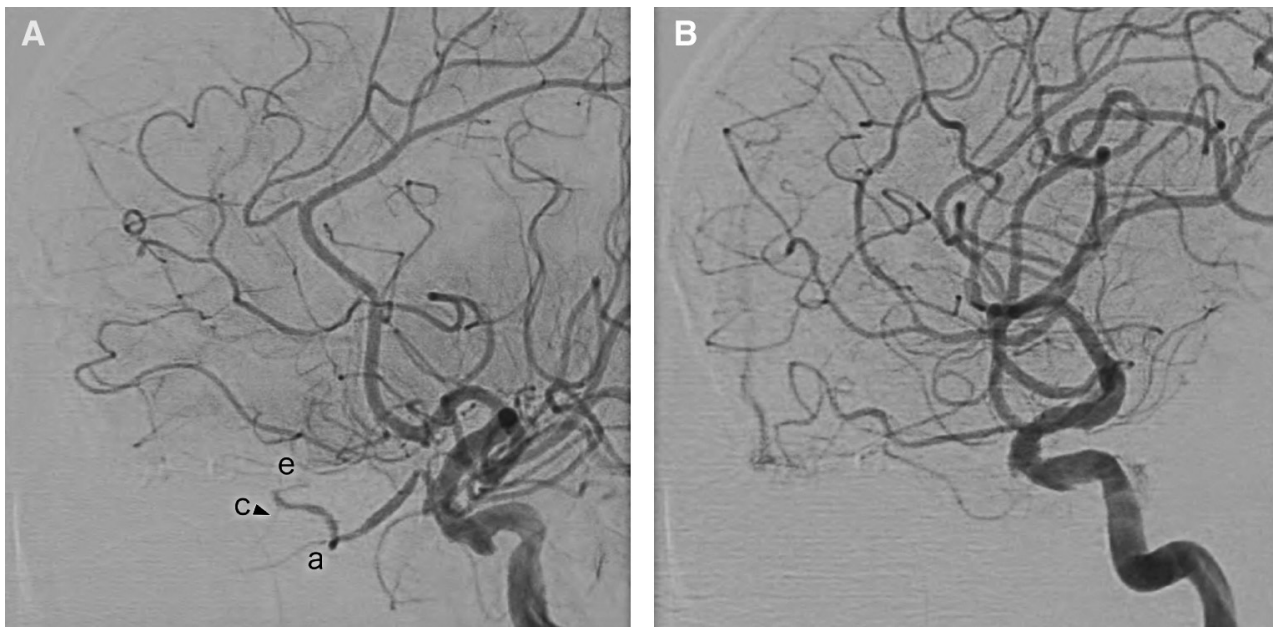

Fig. 4 Postoperative internal carotid angiography. (A) Right internal carotid angiography (lateral view). The periphery to the bifurcation of the PEA was occluded. (a) Central retinal artery/posterior ciliary artery, (c) inferior medial muscular branch (black arrow), and (e) PEA (white arrow head). (B) Left internal carotid angiography (lateral view). The shunt (small volume) remained. PEA: posterior ethmoidal artery

A

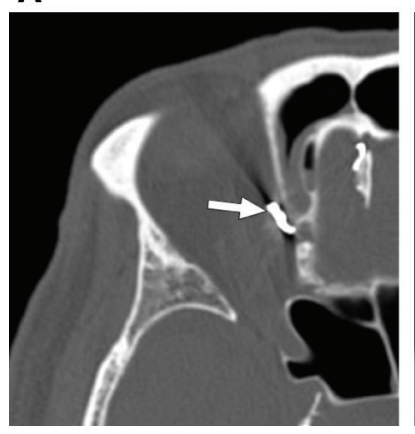

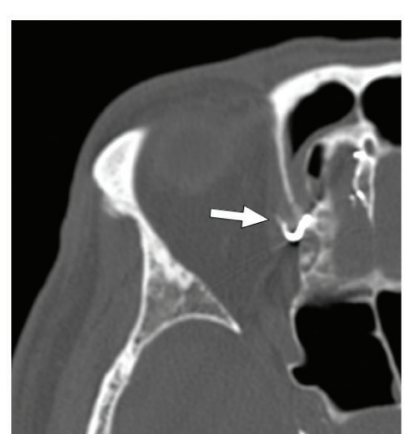

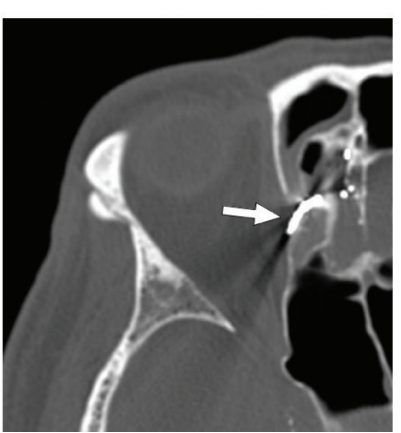

B

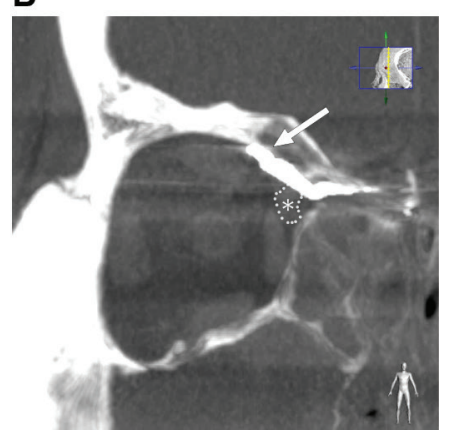

Fig. 5 (A). Cephalic CT: an Onyx cast (arrow) penetrating the anterior ethmoidal foramen from the right orbit was observed. (B) Coronal section on cone-beam CT: the course of the Onyx cast (arrow) in the OphA involved the right intraorbital superior oblique muscle ( ${ }^{*}$ in the dotted line). CT: computed tomography; OphA: ophthalmic artery

possibility of trochlear nerve paralysis, the oral administration of mecobalamin $(1500 \mathrm{mg} /$ day $)$ was started. The symptoms reduced and disappeared 1 month after the procedure. The Hess test results got normalized (Fig. 6). After 5 months, angiography confirmed that the dAVF remained, but there was no exacerbation.

\section{Discussion}

\section{Anterior cranial fossa dAVF}

According to an epidemiological survey in Japan (JR-NET2), $13(1.2 \%)$ of 1075 patients with dAVF had anterior cranial fossa dAVF. ${ }^{4}$

Treatment options include surgical amputation of a drainage route between the dura mater and the cortical vein under craniotomy and TAE from the feeding artery.
Recently, there have been case reports of transvenous embolization via drainage veins such as SSS. $\left.{ }^{5}\right)$

In the present case, TAE was selected based on the patient's age, general condition, and his request.

As complication, the incidence of visual disturbance related to embolization through the OphA is reportedly $0 \%-25 \%{ }^{1-3,6)}$ Even when performing a provocation test, there may be false-positive or false-negative reactions; therefore, if there is a possibility of reflux, the use of liquid embolic substances or small-diameter particles should be avoided. ${ }^{2,6)}$ According to the review of endovascular treatments for anterior cranial fossa dAVF, TAE was performed for 36 of 48 patients, and a trans-AEA route was adopted in 28 (77.8\%). After TAE, complete occlusion was achieved in 32 patients $(88.9 \%)$. As complications, asymptomatic excessive reflux of Onyx to the proximal ocular artery and 

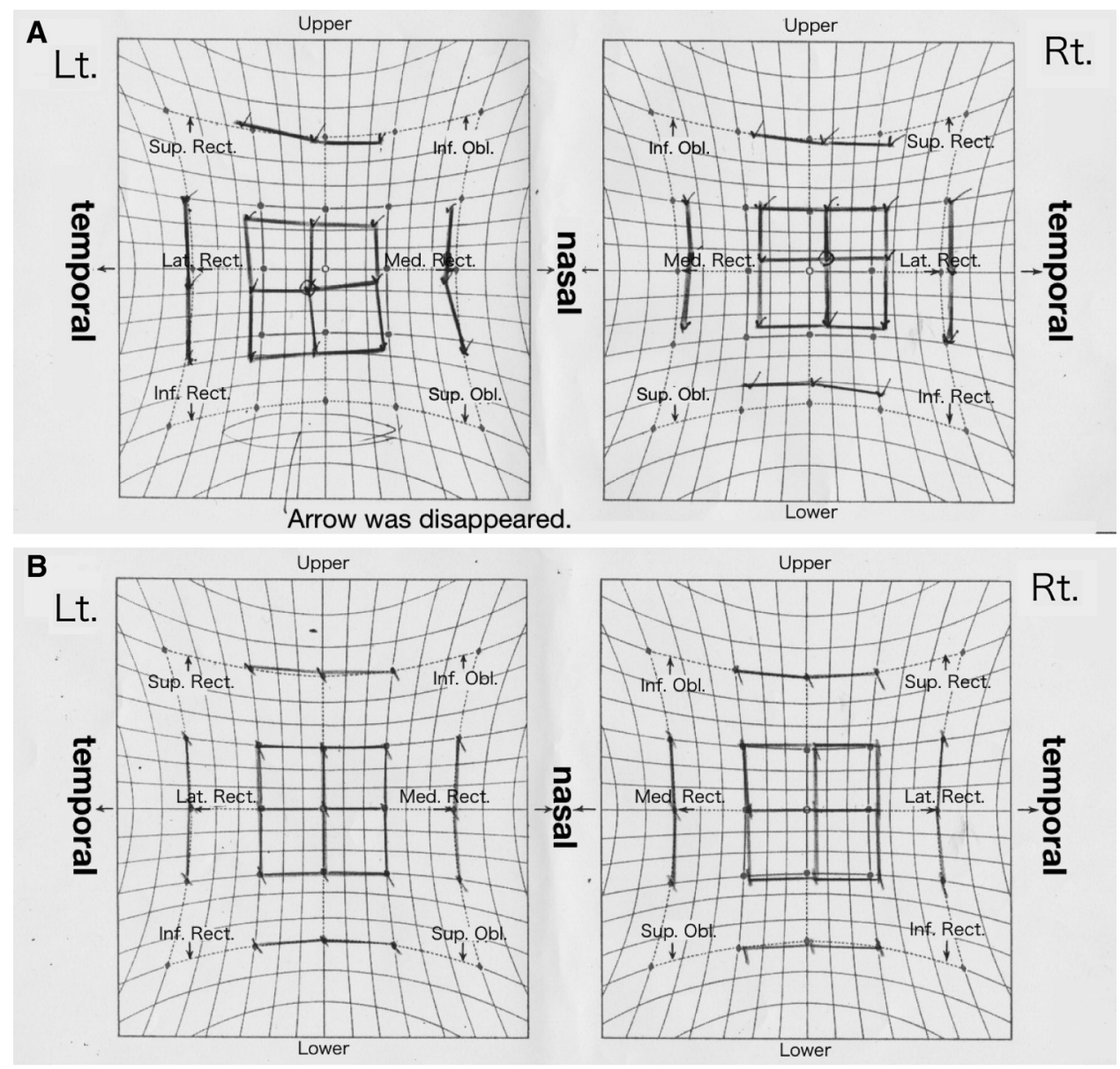

Fig. 6 Hess tests immediately after surgery and after the disappearance of symptoms (2 months after surgery). (A) Immediately after surgery: right superior oblique muscle disorder was noted. (B) Two months after surgery: it was normalized.

difficulty in catheter removal (four patients, $11.1 \%$ ) were observed. There was no case with visual disturbance reported in the review. ${ }^{5}$

Among embolic agents, Onyx is easier to handle than n-butyl-2-cyanoacrylate (NBCA); it can be infused in a large amount at one time and its permeability into the shunt is high. Therefore, the cure rate is high and the incidence of complications is low. Recently, many studies have reported the usefulness of Onyx for dAVFs. On the other hand, when adopting the plug and push method, large volume of Onyx may reflux back to the proximal side through the repeated "push" motions.? Physicians must observe the reflux of onyx carefully during the injection and be familiar with vascular anatomy.

\section{Etiology of ocular movement impairment}

To our knowledge, this is the first report of ocular movement impairment related to embolization through the AEA. In our case, the Bielschowsky's sign was observed. This sign suggests two etiological factors: (1) trochlear nerve disorder and (2) superior oblique muscle disorder. The respective factors are discussed below.

(1) Trochlear nerve disorder

In the present case, Onyx was infused through the periphery of the AEA using the plug and push method. It refluxed back to the third portion of the OphA.

The AEA branch originates from the third portion of the OphA below the superior oblique muscle, and passes into the dura mater around the cribriform plate via the anterior ethmoidal foramen. ${ }^{8)}$ Therefore, this branch frequently feeds anterior cranial fossa dAVF.

The trochlear nerve is originated from the midbrain, and then forms the cisternal segment, intercavernous segment, and intraorbital segment through the superior orbital fissure laterally, and enters into the orbit, reaching the posterior third of the lateral margin of the superior oblique muscle with the anterior medial direction. ${ }^{9)}$ In the intraorbital 
segment, in $60 \%$ of cases, the 4 th nerve crosses with the PEA, ${ }^{10)}$ and nutrition is supplied from the terminal branch of the OphA or AEA. ${ }^{11)}$ Therefore, in the present case, a trochlear nerve-nourishing blood vessel may have been embolized.

(2) Superior oblique muscle disorder

Heyreh et al. examined the course of the intraorbital OphA, using 61 orbits of cadavers, and reported that a superior oblique muscle-nourishing blood vessel had branched from the third portion of the OphA, AEA, or PEA. ${ }^{12)}$ Erdogmus et al. ${ }^{13)}$ found that the right superior oblique muscle-nourishing vessels included the bend of the OphA (36.84\%), PEA (26.31\%), inferolateral muscular trunk (5.26\%), second portion of the OphA (5.26\%), AEA $(5.26 \%)$, and supra-orbital artery $(5.26 \%)$.

In the present case, the third portion of the OphA and AEA were occluded, indicating the risk of paralysis of the superior oblique muscle itself.

Ocular movement impairment in this case may have been related to occlusion of a trochlear nerve-nourishing vessel. However, the occluded OphA and AEA were also superior oblique muscle-nourishing vessels; therefore, we cannot exclude the possibility of ischemia of the superior oblique muscle.

\section{Dimethyl sulfoxide-related neurotoxicity}

Previous studies suggested that embolization using Onyx induced neurotoxicity related to dimethyl sulfoxide (DMSO).

In the experiment involving the infusion of a liquid embolic substance into the rabbit subarachnoid cavity, inflammatory responses to Onyx or NBCA and nerve tissue toxicity were confirmed. However, these reactions to Onyx were less marked than those to NBCA. ${ }^{14)}$

Baker et al. reported that DMSO was infused through the rat ICA, followed by evaluating changes in the hippocampu and intracranial arteries. When DMSO was infused rapidly, vasospasm was observed. However, when it was infused slowly, there was no influence on the nerve cells, bloodbrain barrier (BBB), or blood vessels even at a large volume. ${ }^{15)}$ Murayama ${ }^{16)}$ also reported complications associated with vasospasm or vascular endothelial injury related to the rapid infusion of DMSO.

A previous study histopathologically investigated 32 patients with arteriovenous malformation (AVM) who underwent embolization using Onyx or NBCA (Onyx: 22 patients, NBCA: 12), and found no extravascular leakage of the embolic substance. ${ }^{17}$ )

DMSO or Onyx into the subarachnoid cavity has neurotoxicity. Although rapid infusion into blood vessels may cause vasospasm, BBB destruction or extravascular leakage is rare. As there was no extravascular leakage in the present case, the possibility of neurotoxicity of Onyx was probably excluded.

\section{Conclusion}

We report a rare case in which paralysis of the superior oblique muscle developed after embolization using Onyx through the AEA. Even embolization through the periphery to the third portion of the OphA may cause extraocular muscle paralysis, leading to ocular movement impairment; therefore, caution is needed.

\section{Acknowledgments}

We deeply appreciate Michihiro Tanaka for his advice about the neuroanatomy, and Tomoyuki Tsumoto and Yoshikazu Matsuda for the assistance of making the manuscript.

\section{Disclosure Statement}

We declare no conflict of interest.

\section{References}

1) Terada $T$, Kinoshita $Y$, Yokote H, et al: Preoperative embolization of meningiomas fed by ophthalmic branch arteries. Surg Neurol 1996; 45: 161-166.

2) Trivelatto F, Nakiri GS, Manisor M, et al: Preoperative onyx embolization of meningiomas fed by the ophthalmic artery: a case series. AJNR Am J Neuroradiol 2011; 32: 1762-1766.

3) Alvarez H, Rodesch G, Garcia-Monaco R, et al: Embolisation of the ophthalmic artery branches distal to its visual supply. Surg Radiol Anat 1990; 12: 293-297.

4) Hiramatsu M, Sugiu K, Hishikawa T, et al: Epidemiology of dural arteriovenous fistula in Japan: analysis of Japanese Registry of Neuroendovascular Therapy (JR-NET2). Neurol Med Chir (Tokyo) 2014; 54: 63-71.

5) $\mathrm{Xu} \mathrm{K}, \mathrm{Ji} \mathrm{T}$, Li C, et al: Current status of endovascular treatment for dural arteriovenous fistulae in the anterior cranial fossa: a systematic literature review. Int J Med Sci 2019; 16: 203-211.

6) Lefkowitz M, Giannotta SL, Hieshima G, et al: Embolization of neurosurgical lesions involving the ophthalmic artery. Neurosurgery 1998; 43: 1298-1303.

7) Li C, Wu Z, Yang $X$, et al: Transarterial treatment with Onyx of Cognard type IV anterior cranial fossa dural arteriovenous fistulas. J Neurointerv Surg 2014; 6: 115-120. 
8) Rhoton AL, Jr., Matsushima T, Inoue T et al: This is a translation of RHOTON-cranial anatomy and surgical approaches, Originally Published in 2003. Tokyo: Nankodo; 2017.

9) Rene C. Update on orbital anatomy. Eye (Lond) 2006; 20 : 1119-1129.

10) Villain M, Segnarbieux F, Bonnel F, et al: The trochlear nerve: anatomy by microdissection. Surg Radiol Anat 1993; 15: 169-173.

11) Iaconetta $\mathrm{G}$, de Notaris $\mathrm{M}$, Benet $\mathrm{A}$, et al: The trochlear nerve: microanatomic and endoscopic study. Neurosurg Rev 2013; 36: 227-228.

12) Hayreh SS, Dass R: The ophthalmic artery: II. Intra-orbital course. Br J Ophthalmol 1962; 46: 165-185.

13) Erdogmus S, Govsa F: Arterial vascularization of the extraocular muscles on its importance for orbital approaches. J Craniofac Surg 2007; 18: 1125-1132.
14) Bakar B, Oruckaptan HH, Hazer BD, et al: Evaluation of the toxicity of onyx compared with n-butyl 2-cyanoacrylate in the subarachnoid space of a rabbit model: an experimental research. Neuroradiology 2010; 52: 125-134.

15) Bakar B, Kose EA, Sonal S, et al: Evaluation of the neurotoxicity of DMSO infused into the carotid artery of rat. Injury 2012; 43: 315-322.

16) Murayama $Y$, Viñuela F, Ulhoa A, et al: Nonadhesive liquid embolic agent for cerebral arteriovenous malformations: preliminary histopathological studies in swine rete mirabile. Neurosurgery 1998; 43: 11641175.

17) Natarajan SK, Born D, Ghodke B, et al: Histopathological changes in brain arteriovenous malformations after embolization using Onyx or N-butyl cyanoacrylate. J Neurosurg 2009; 111: 105-113. 